\title{
Study of coexistence between indoor LTE femtocell and outdoor-to-indoor DVB-T2-Lite reception in a shared frequency band
}

\author{
Ladislav Polak, Lukas Klozar, Ondrej Kaller, Jiri Sebesta, Martin Slanina and Tomas Kratochvil
}

\begin{abstract}
Nowadays, the demand for high-quality multimedia services (video, audio, image, and data) is rapidly increasing. The Digital Video Broadcasting - terrestrial (DVB-T) standard, its second-generation version (DVB-T2), and the Long-Term Evolution (LTE) standard are the most promising systems to fulfill the demand for advanced multimedia services (e.g., high-definition image and video quality), especially in Europe. However, LTE mobile services can operate in a part of the UHF band allocated to DVB-T/T2 TV services previously. The main purpose of this work is to explore the possible coexistences of DVB-T2-Lite and LTE systems in the same shared frequency band (co-channel coexistence) under outdoor-to-indoor and indoor reception conditions. Furthermore, an applicable method for evaluating coexistence scenarios between both systems is shown with a particular example. These coexistence scenarios can be noncritical and critical. In the first case, both systems can coexist without significant performance degradation. In the second one, a partial or full loss of DVB-T2-Lite and/or LTE signals can occur. We consider an indoor LTE femtocell and outdoor-to-indoor DVB-T2-Lite signal reception in a frequency band from 791 up to $821 \mathrm{MHz}$. Simulations of combined indoor and outdoor signal propagation are performed in MATLAB using 3rd Generation Partnership Project (3GPP) channel models, separately for both DVB-T2-Lite and LTE systems. Correctness of path loss simulation results is verified by measurements. Afterwards, an appropriate linear model is proposed which enables to evaluate the impact of coexistence on performance of both systems in outdoor-to-indoor and indoor-to-indoor reception scenarios. The results are related to an actual location in the building and are presented in floor plans. The floor plans include different coexistence conditions (different power imbalance and different amount of overlay of the radio channels). Service availability of both systems is verified again by measurements. The resulting maps help better understand the effect of coexistence on achievable system performance under different indoor/outdoor reception situations considering real transmission conditions.
\end{abstract}

Keywords: Channel model; Coexistence; DVB-T2-Lite; Indoor and outdoor-to-indoor propagation; LTE femtocell; Path loss; QEF; EVM; CQI; RF measurement

\section{Introduction}

Advanced wireless communication systems can provide users with any type of multimedia. Thanks to this, the idea to 'connect, upload, download, share and transfer anything at anytime and anywhere' is not a futuristic vision $[1,2]$. From the viewpoint of service providers, efficient usage of limited resources in the radio frequency (RF) spectrum is one of the biggest challenges. Hence, the increasing density of wireless networks and the

\footnotetext{
* Correspondence: polakl@feec.vutbr.cz

Department of Radio Electronics, SIX Research Center, Brno University of Technology, Technicka 3082/12, 61600 Brno, Czech Republic
}

increasing volume of user equipment (UE) terminals in use escalate the risk of unwanted coexistence scenarios $[3,4]$.

In the near future, the next-generation digital terrestrial television broadcasting (DVB-T2/T2-Lite) and Long-Term Evolution (LTE) systems will be deployed to provide multimedia services for mobile and portable scenarios, mainly in Europe. DVB-T2-Lite [5-8] is a new profile which was added to the DVB-T2 system specification in April 2012. This subset within DVB-T2 is very perspective for mobile and portable TV broadcasting as it is designed to support 
low-capacity applications for advanced handheld receivers [9]. It is based on the same core of technologies as the DVB-T2 standard but uses only a limited number of available modes. By avoiding the modes, which require the most computational power and memory [6], the necessary complexity of T2-Lite-only receivers is reduced. DVB-T2-Lite, compared to the first-generation DVB-T/ $\mathrm{H}$ [10], can support TV content delivery with higher flexibility. Moreover, it can operate in VHF (from 174 up to $230 \mathrm{MHz}$ ) and UHF (from 470 up to $870 \mathrm{MHz}$ ) bands, allocated earlier for DVB-T/H. From the viewpoint of system flexibility, spectral efficiency, and available transmission scenarios, DVB-T2-Lite is the system of choice for the next-generation terrestrial mobile and portable digital TV broadcasting.

Third Generation Partnership Project (3GPP) LTE [11-13] technology brings a new concept, based on the Orthogonal Frequency Division Multiple Access (OFDMA), into mobile communications. LTE supports high data rates and flexible system configuration in order to adapt transmission parameters to the actual state of a radio link. LTE architecture involves a specific type of cells called femtocells. These short ranges, mainly indoor cells, improve coverage in desired areas, especially buildings. Femtocells are served by a special type of base station called Home eNodeB (HeNB). LTE can exploit the same UHF frequency bands which are already available for existing 2G/3G networks (e.g., bands: 800, 900, 1,800, and 2,600 MHz). Moreover, additional ranges (from 2.5 up to $2.7 \mathrm{GHz}$ ) and the $700-\mathrm{MHz}$ band are also allocated for LTE usage. The European Union decided to harmonize the ' $800 \mathrm{MHz}$ band' in favor of the LTE services, starting from January 2013 [4]. Consequently, DVB-T/T2 and LTE services can occupy either the same or adjacent frequency spectrum. As a result, unwanted coexistence between DVB-T/T2 and LTE services can occur $[4,14]$.

This work deals with the study of possible co-channel coexistence between DVB-T2-Lite (outdoor-to-indoor reception) and LTE services (provided by the femtocell) under fixed indoor reception conditions.

The paper is organized as follows. An overview of related work in the field of different wireless standards' coexistence, especially DVB and LTE, is presented in Section 2. This section also includes a detailed list of aims and contributions of this work. A description of the explored coexistence scenario and the considered DVB-T2-Lite system parameters are presented in Section 3. Section 4 contains a description of the applied simulation method and the proposed measurement testbed together with its detailed setup. Results obtained from simulation and measurements are presented and discussed in Section 5. Finally, Section 6 concludes the paper.

\section{Related works}

Undesirable interactions between similar or different kinds of wireless communication systems, operating in adjacent or shared frequency bands, are not a new phenomenon [3,15-18]. The exploration, monitoring, measurement, and possible suppression of interferences are a hot topic. This fact is also evidenced by many published studies available. Authors of [19] studied the possible inter-band interferences between UMTS and GSM systems. In another work [20], the coexistence between advanced wireless systems and International Mobile Telecommunication-Advanced (IMT-A) services is explored. Different kinds of coexistence scenarios in LTE networks are analyzed in [21-23]. Possible methods to mitigate or suppress interferences from coexistence between two different wireless systems are outlined in [24-26].

In the last decade, researchers' attention has been devoted to the study of different coexistence scenarios between the DVB-T/T2 and LTE/LTE-A standards.

Table 1 summarizes the previously explored coexistence scenarios between such systems. From the presented works, it is clearly seen that many times the researchers use either only simulation tools or only different measuring methods to explore the coexistence scenarios. Furthermore, in most works, a scenario is considered in which macrocells are used to provide LTE service coverage, coexisting with DVB-T2-Lite services in the same or adjacent frequency band. The main aim of this research article is to explore the interaction of DVB-T2-Lite and LTE in a shared frequency band, such that femtocells (HeNB) are used to provide LTE indoor coverage. Attention is devoted to availability monitoring of DVB-T2-Lite and LTE services in different locations under fixed indoor reception conditions. For this purpose, an appropriate simulation model is proposed and verified by measurement. Based on these results, noncritical (both DVB-T2-Lite and LTE system working) and critical (partial or full loss of DVB-T2-Lite and/or LTE signals) coexistence scenarios can be identified and the general conclusions are outlined. To the best of our knowledge, no similar exploration in this form has been presented in any scientific or technical paper so far.

\section{Considered coexistence scenario}

The investigated coexistence scenario between the DVB-T/ $\mathrm{T} 2$ and LTE RF signals in the fixed indoor transmission scenario is shown in Figure 1. The main system parameters of DVB-T2-Lite and LTE systems, considered in this work, can be found in Table 2. The DVB-T2-Lite TV signal is broadcast in a single frequency network (SFN) at a center frequency of 794 MHz and received by UE1 in a building. In the same building, LTE femtocells are deployed and the HeNB provides mobile connectivity in a channel belonging to Band 20 (from 791 up to $821 \mathrm{MHz}$ ). A user of UE2 
Table 1 Comparison of explored coexistence scenarios between DVB-T/T2 and LTE systems

\begin{tabular}{|c|c|c|c|c|}
\hline Reference & Coexistence scenario (TV broadcast scenario) & Type of interference & Results & Evaluation parameters \\
\hline [42] & DVB-T vs. LTE (fixed) & Mutual co-channel & Simulation & SNR, SINR, QOS \\
\hline [43] & LTE vs. DVB-T (fixed, portable) & Adjacent channel & Simulation & $P R, C R$ \\
\hline [44] & DVB-T vs. LTE (fixed) & Adjacent channel & Simulation & $\mathrm{BER}, \mathrm{PF}, \mathrm{PR}$ \\
\hline [45] & DVB-T vs. LTE (fixed) & Intersystem & Simulation & IPL, spectral overlap \\
\hline [27] & LTE-A vs. DVB-T (fixed) & Intersystem & Simulation & $\mathrm{ADL}, \mathrm{FO}$ \\
\hline [46] & LTE vs. DVB-T (fixed) & Intersystem (co-channel) & Measurement & Data throughput, RSRQ \\
\hline [47] & DVB-T vs. LTE (fixed) & Co-channel & Simulation & $\mathrm{l} / \mathrm{N}, \mathrm{C} /(\mathrm{N}+\mathrm{l})$ \\
\hline [48] & DVB-T/H vs. LTE (fixed) & Co-channel & Measurement & SSIM, QEF, SIR \\
\hline [30] & LTE vs. DVB-T2-Lite (mobile) & Adjacent channel & Measurement & SDR, QEF, EVM, MER \\
\hline [31] & DVB-T/T2 vs. LTE (partly mobile, fixed) & Co-channel and adjacent channel & Measurement & SDR, BER, EVM, MER \\
\hline This study & DVB-T2-Lite vs. LTE (fixed) & Co-channel (partial overlapping) & Simulation/Measurement & QEF, partly CQI, EVM \\
\hline
\end{tabular}

Abbreviations: $\mathrm{ADL}$, antenna discrimination loss; $\mathrm{PER}$, packet error ratio; $\mathrm{BER}$, bit error ratio; $\mathrm{PF}$, picture failure; $\mathrm{Cl}$, carrier-to-interference ratio; $\mathrm{PR}$, protection ratio; $\mathrm{CR}$, correction factor; $\mathrm{QEF}$, quasi error-free; $\mathrm{CQI}$, channel quality indicator; QoS, quality-of-service; $\mathrm{C} /(\mathrm{N}+\mathrm{l})$, carrier-to-noise+interference ratio; $\mathrm{RSRQ}$, reference signal received quality; EVM, error vector magnitude; SDR, spectral density ratio; FO, frequency offset; SIR, signal-to-interference ratio; IPL, interference power level; SINR, SIR plus noise-ratio; I/N, interference associated to new sources; SNR, signal-to-noise ratio; MER, modulation error ratio; SSIM, structural similarity.

establishes connection with HeNB at downlink frequency band from 795 (797.2 $\mathrm{MHz})$ to $805 \mathrm{MHz}(817.2 \mathrm{MHz})$. We consider that the bandwidth of the LTE signal is 10 or $20 \mathrm{MHz}$, and intersystem frequency overlapping is from 0.8 up to $3 \mathrm{MHz}$. Consequently, coexistence between HeNB (supporting 3GPP LTE Release 9) and DVB-T2-Lite system can occur. As a specific type of coexistence, a partial overlapping scenario is assumed. It means that the channel of the interferer (in this case LTE) partially overlaps with the channel of the victim (in this case DVB-T2Lite) [27]. It is assumed that both UEs are stationary.

\section{Simulation and measurement setup}

In this section, the simulation method, used to explore the coexistence of digital TV and mobile RF signals under outdoor-to-indoor and indoor-to-indoor conditions, is presented. Furthermore, the proposed measurement testbed and its setup, used in this work, are introduced. The simulation and measurement campaign consists of the following:

1. Simulation (propagation loss) and measurement of LTE performance in different locations (indoor and outdoor environment);

2. Simulation (propagation loss) and measurement of DVB-T2-Lite performance in different locations (indoor and outdoor environment);

3. Simulation and measurement of simultaneous transmission (signal propagation) of both LTE and DVB-T2-Lite RF signals in order to evaluate the

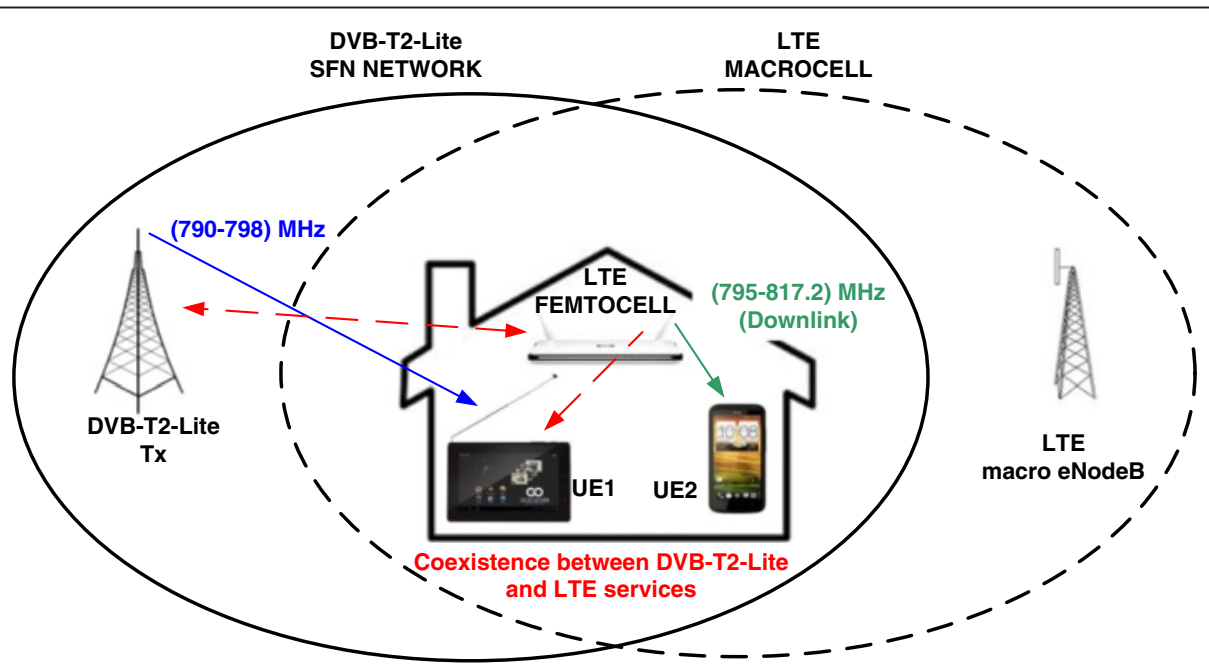

Figure 1 Unwanted coexistences between DVB-T2-Lite and LTE services at fixed indoor transmission scenario. Supposed scenario where LTE femtocell is indoors and DVB-T2-Lite signal penetrates from outdoor transmitter and affects performance. 


\begin{tabular}{|c|c|c|}
\hline Definition of parameters & DVB-T2-Lite & LTE (Release 9) \\
\hline Type of FEC scheme & $\mathrm{BCH}$ and LDPC & Turbo \\
\hline FEC code rate & $2 / 3$ & $1 / 3$ \\
\hline \multirow[t]{3}{*}{ Type of modulation } & 16QAM & QPSK \\
\hline & & 16QAM \\
\hline & & 64QAM \\
\hline Constellation rotation & No & - \\
\hline \multirow[t]{2}{*}{ IFFT size } & $2,048(2 K)$ & 1,024 (10 MHz) \\
\hline & & 2,048 (20 MHz) \\
\hline Type of PP pattern & PP2 & - \\
\hline Guard interval duration & $28 \mu \mathrm{s}$ & $4.7 \mu \mathrm{s}$ \\
\hline Transmission mode & SISO & SISO \\
\hline Carrier frequency (MHz) & 794 & Downlink $(791 \div 821)$ \\
\hline Channel bandwidth & $8 \mathrm{MHz}$ & $10 \mathrm{MHz}, 20 \mathrm{MHz}$ \\
\hline RF power & (0.1 to 5) W & (0.01 to 0.06$) \mathrm{W}$ \\
\hline Channel environment & $\begin{array}{l}\text { Outdoor-to- } \\
\text { indoor }\end{array}$ & $\begin{array}{l}\text { Indoor (indoor-to- } \\
\text { outdoor) }\end{array}$ \\
\hline FEC decoding method & 1D LLR [6] & Max Log-map \\
\hline $\begin{array}{l}\text { Tx antenna height (m) } \\
\text { (above floor) }\end{array}$ & 2 & 1 \\
\hline $\begin{array}{l}\text { Rx antenna height (m) } \\
\text { (above floor) }\end{array}$ & 1 & 1 \\
\hline LTE user equipment & - & $\begin{array}{l}\text { Huawei e389u-15 (LTE UE } \\
\text { category 3) }\end{array}$ \\
\hline
\end{tabular}

$\mathrm{BCH}$, Bose-Chaudhuri-Hocquenghem; LLR, log likelihood ratio; FEC, forward error correction; PP, pilot pattern; IFFT, inverse fast Fourier transform; SISO, single-input single-output; LDPC, low-density parity-check.

influence of coexistence on the performance of both systems (on physical layer (PHY) level); and

4. Identification of the noncritical (both systems can coexist) and critical (partial or full loss of DVB-T2-Lite and LTE signal) coexistence scenarios for both systems.

\subsection{Simulation setup}

The considered coexistence scenario was briefly outlined in the previous section. In this work, we assume that transmitters and receivers are located on the seventh floor (the top floor) in the building of Brno University of Technology (BUT), Faculty of Electrical Engineering and Communications (FEEC) in Brno. Laboratories of Digital TV Technology and Radio Communications, and Mobile Communications of the Department of Radio Electronics (DREL) are located on this floor. The floor plan of the seventh floor is shown in Figure 2. Approximate dimensions of the floor are $50 \times 25 \mathrm{~m}$. The HeNB is located in the Laboratory of Mobile Communication Systems (room 7107), and the DVB-T2-Lite transmitter is located outdoor on the terrace.
The whole simulation model is realized in MATLAB. Propagation of the LTE and DVB-T2-Lite RF signals are simulated separately. The simulation of separate propagation loss of LTE and DVB-T2-Lite RF signals will be used as the reference (no coexistence).

The simulation model consists of three main parts for both LTE and DVB-T systems. The first part represents the simulation of a link budget, according to the 3GPP recommendation for system level simulations $[28,29]$ for both coexisting systems. Signal strength in the receiver can be expressed as follows:

$$
P_{\mathrm{RX}}=P_{\mathrm{TX}}-L_{\mathrm{TXC}}+G_{\mathrm{TXA}}-P L+G_{\mathrm{RXA}}-L_{\mathrm{RXC}}
$$

where $P_{\mathrm{TX}}$ is transmitter power, $L_{\mathrm{TXC}}$ are wiring losses, $G_{\mathrm{TXA}}$ is transmitting antenna gain, $G_{\mathrm{RXA}}$ is receiving antenna gain, $L_{\mathrm{RXC}}$ are wiring losses, and finally, $P_{\mathrm{RX}}$ is received signal level. Path losses in wireless transmission are denoted as $P L$ (for details see Equation 3). Value of $P_{\mathrm{TX}}$ is known from the transmitter setup. Values of $L_{\mathrm{TXC}}, L_{\mathrm{RXC}}, G_{\mathrm{TXA}}$, and $G_{\mathrm{RXA}}$ are constants depending on the used equipment (for details see Subsection 4.2). The second part represents the validation of obtained results from the simulation according to the performed measurement and their interpretation in a map. Details are in Subsections 4.1 and 4.2. The last part compares power imbalance of tested radio channels and computed achievable performance of both systems in certain locations. Details are given in Section 5.

The propagation scenario of the LTE RF signal in femtocell involves indoor-to-indoor line-of-sight (LOS) propagation for the same room where HeNB is located (room 7107) and non-LOS (NLOS) for other indoor locations. Path losses are modeled according to the 3GPP recommendation for indoor LTE femtocell as described in [28], denoted as UE to HeNB, where UE is inside the same building as HeNB. In order to model indoor-to-outdoor propagation from the HeNB to the measurement points on the terrace, the original equation was extended with outdoor wall penetration loss. On the other hand, the recommendations in [28] are generally valid for frequencies around $2 \mathrm{GHz}$, but we exploit an $800-\mathrm{MHz}$ band in this study. Therefore, it is necessary to perform a correction as described in [29]. This correction defines the correction factor for $800 \mathrm{MHz}$ as follows:

$$
P L_{\mathrm{COR}}=20 \log _{10}\left(f_{c}\right)
$$

where $f_{c}$ is carrier frequency in $\mathrm{MHz}$.

The resulting path loss equation is:

$$
\begin{aligned}
\mathrm{PL}= & 38.46+20 \log (d)+0.7 d_{\text {in }}+\mathrm{LP}_{\text {floor }} \\
& +q L_{\text {INwall }}+n L_{\text {OUTwall }}+\mathrm{PL}_{\mathrm{COR}}
\end{aligned}
$$

where $d$ is the distance between the HeNB and the UE, $d_{\text {in }}$ is indoor propagation distance, $L P_{\text {floor }}$ is penetration 


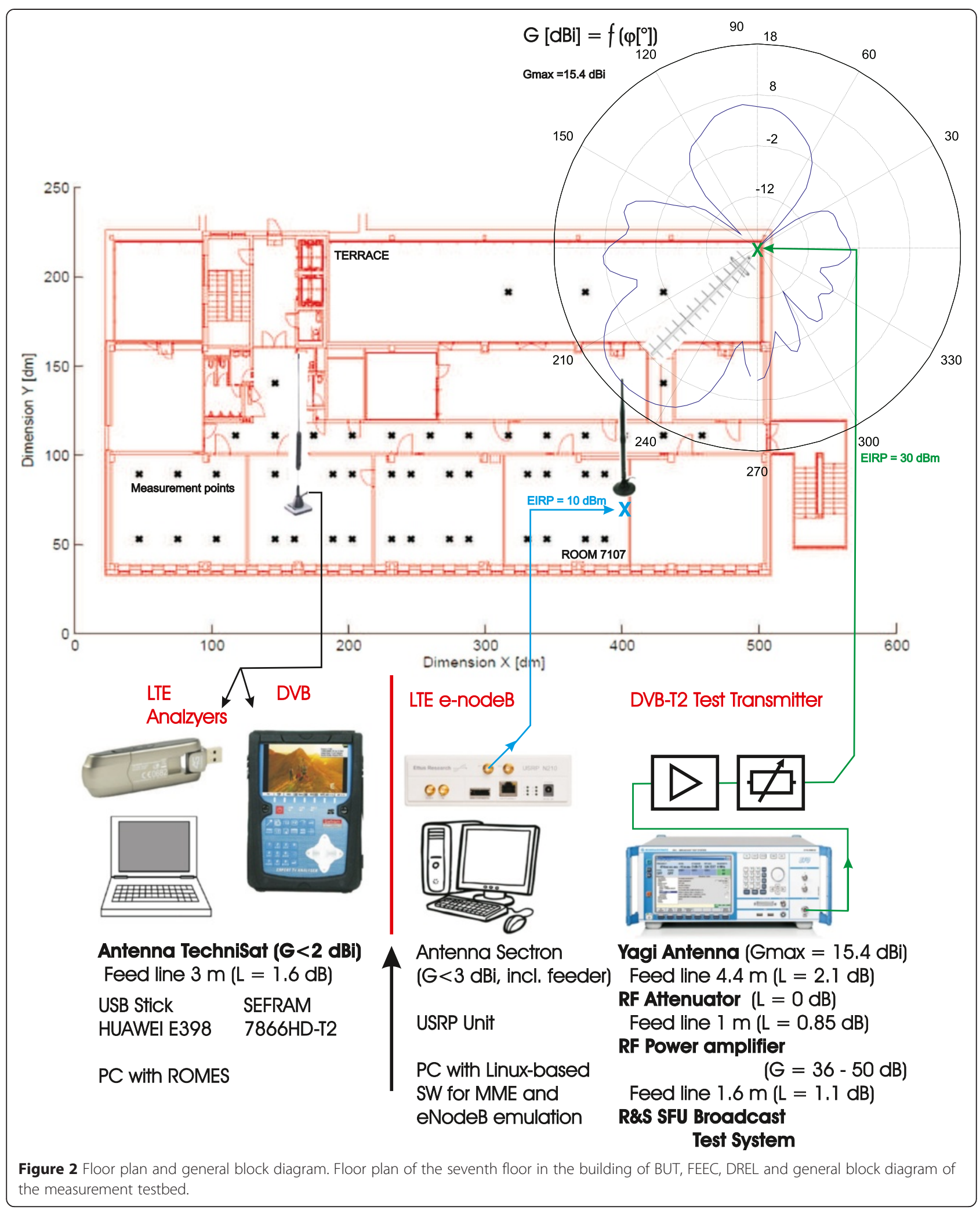

loss due to propagation through the floor (it is equal to zero because a single-floor propagation scenario is assumed), parameter $q$ is the number of indoor walls separating the transmitter and receiver, $L_{\mathrm{INwall}}$ is the penetration loss due to walls inside the building, $n$ is the number of outside walls, $L_{\text {OUTwall }}$ is the penetration loss 
of the exterior wall, and $P L_{\mathrm{COR}}$ is the frequency correction factor as defined in [29] and shown in Equation 2. In our case $d_{\text {in }}=d, L P_{\text {floor }}=0$ (single floor), $q>0$ (in the case of the NLOS scenario), $L_{\mathrm{INwall}}=5 \mathrm{~dB}, n$ is between 0 and 5 (depending on the concrete position on the floor) and $L_{\text {OUTwall }}=10 \mathrm{~dB}$.

The propagation scenario between the DVB-T2-Lite transmitter and TV receiver is considered as outdoor-toindoor urban femtocell propagation, where the UE is outside as described in [29]. The DVB-T2-Lite RF signal attenuation with frequency correction can be calculated similarly to Equation 3 as:

$$
\begin{aligned}
\mathrm{PL}= & \max \left(15.3+37.6 \log _{10}(d), 38.46+20 \log _{10}(d)\right) \\
& +0.7 d_{\text {in }}+L P_{\text {floor }}+q L_{\text {INwall }}+n L_{\text {OUTwall }}+\mathrm{PL}_{\mathrm{COR}}
\end{aligned}
$$

where all variables have the same meaning as in Equation 3.

No fading was included in the data displayed in Figures 3 and 4, however, both fast fading and shadowing were computed according to recommendations in [29].

Figures 3 and 4 show the results of LTE and DVB-T2Lite radio signal propagation obtained from simulation, respectively. System parameters determined according to the simulation results prove accessibility of wireless services in all tested locations.

Path loss model data provides the basis for coexistence simulations. We have provided a detailed description of LTE and DVB-T2-Lite coexistence in our previous works [30] and [31]. Based on data collected from the mentioned measurements, we made a dense description (linear model) of coexistence. There are two types of input parameters for the models: global and local. The global parameters are mainly represented by the settings of both systems' PHY, such as modulation used in DVBT2-Lite, inverse fast Fourier transform (IFFT) size, and the Forward Error Correction (FEC) code rate of both systems. Obviously, the overlapping bandwidth is also a global parameter. Local parameters, used as the model input, are mainly local power levels of signals, background noise, and the local fading model employed. These parameters are input into the linear model, which maps them to the Quality-of-Service (QoS) parameters. More details can be found in Subsection 5.1.

\subsection{Measurement setup}

For evaluating the interaction of the described coexistence scenarios between DVB-T2-Lite and LTE RF signals, the same measurement testbed was used as described in our previous works ([30] and [31]). The whole measurement campaign was implemented on the seventh floor of the building of BUT, FEEC, DREL (see Figure 2). The measurement campaign and the basic principle of our measurement method are as follows.
Firstly, the parameters and performance of the 3GPP LTE network are measured in different locations on the seventh floor. At the time of LTE measurement, T2-Lite services were not broadcasted. The HeNB is located in room 7107, and its antennas are placed on top of a table (approximately $1 \mathrm{~m}$ above the floor). The HeNB consists of two main hardware components, namely a PC with the Fedora Linux operating system and universal software radio peripheral (USRP) N210 from Ettus, equipped with an SBX daughter card. The PC runs the commercial software package Amari LTE [32], implementing functions of LTE Mobile Management Entity (MME) and eNB (both are 3GPP LTE Release 9 compliant). A detailed configuration of the LTE network is summarized in Table 2. The receiving UE is Huawei e398-u15 (Huawei, Shenzhen, China) (LTE UE Cat. 3) [33], connected via USB port to a laptop equipped with the Rohde \& Schwarz drive test software ROMES4. For receiving LTE services, the TechniSat Digiflex TT1 mobile antenna (TechniSat, Vulkaneifel, Germany) was used $(G<2 \mathrm{dBi})$. The length of its feed line is $3 \mathrm{~m}$. The UE is connected to an external antenna placed on a wooden cart approximately $1.0 \mathrm{~m}$ above the floor. We set up the connection between UE and HeNB and performed simultaneous full buffer transmissions in uplink and downlink. The measurement was carried out in fixed points distributed on the seventh floor as shown in Figure 2. The receiving antenna was kept still for $2 \mathrm{~min}$ at each measurement point and in each location we have collected approximately 100 samples of each network parameter of interest (including RSS, Channel Quality Indicator (CQI), Error Vector Magnitude (EVM), etc.).

Secondly, we have measured the performance of the DVB-T2-Lite signal in different locations on the seventh floor. At the time of T2-Lite measurement, LTE services were not provided. By using the $R \& S$ single frequency unit (SFU) broadcast test system, an appropriate video transport stream for portable TV scenarios was generated. Then, the DVB-T2-Lite complete system configuration was set up, and the output signal was RF modulated (to the frequency of $794 \mathrm{MHz}$ ). For its amplification, a custom-built RF power amplifier (PA), based on hybrid module Mitsubishi RA20H8087M (Mitsubishi Electric, Tokyo, Japan) [34], was applied. This RF three-stage module is primarily destined for transmitters using FM modulation that operate in the range 806 up to $870 \mathrm{MHz}$, but it may also be applied in linear systems by setting the proper drain quiescent current with externally settable gate voltage. The PA was assembled according to the recommendations of the producers and thoroughly tested. The comprehensive measurement demonstrates that this PA can be used in a wider band, circa from 650 to $900 \mathrm{MHz}$, and can be used in the presented coexistence test. The gain of the PA strongly varies in the introduced frequency range from 36 to $50 \mathrm{~dB}$, but in a 


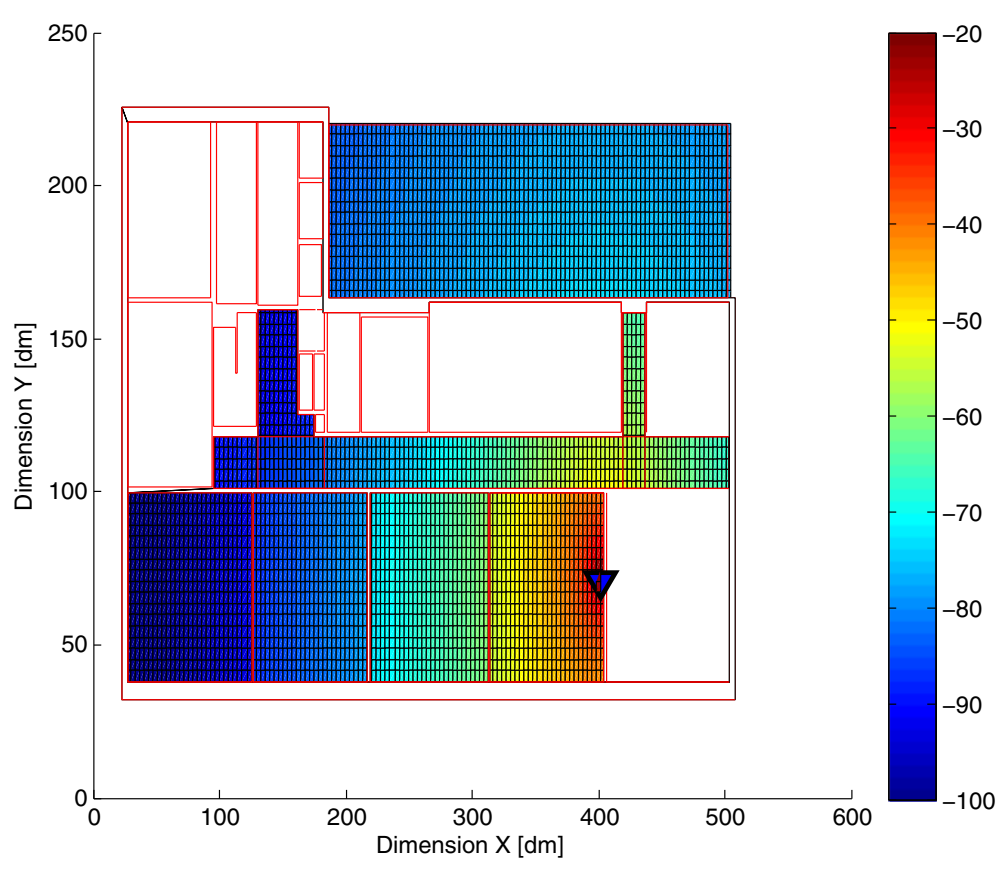

Figure 3 Simulation of LTE RF signal propagation. The HeNB is located in room 7107 (the blue triangle), and network parameters are as described in Table 2. The path loss model was adopted from [28] and extended for the 800-MHz band according to [29] (see Equations 2 and 3). All values are in dBm.

narrow band, the gain is quite stable (max. $1.5 \mathrm{~dB}$ in 10 $\mathrm{MHz}$ bandwidth). The maximum output power of this amplifier is around $30 \mathrm{~W}$. However, we practically used only $1 \mathrm{~W}(5 \mathrm{~W}$ was used for the scenario where power imbalances were equal to $20 \mathrm{~dB}$ ) with quiescent drain current 4. A, gate bias voltage $4.3 \mathrm{~V}$, and supply voltage $13.8 \mathrm{~V}$ to achieve high linearity for reliable application in the mentioned setup. Accordingly, the power efficiency in this setting is only $2 \%$. On the other hand, reaching linearity is the fundamental parameter which needs to be set for minimizing any nonlinear distortion. For the used testing DVB-T2-Lite frequency $(794 \mathrm{MHz})$, the

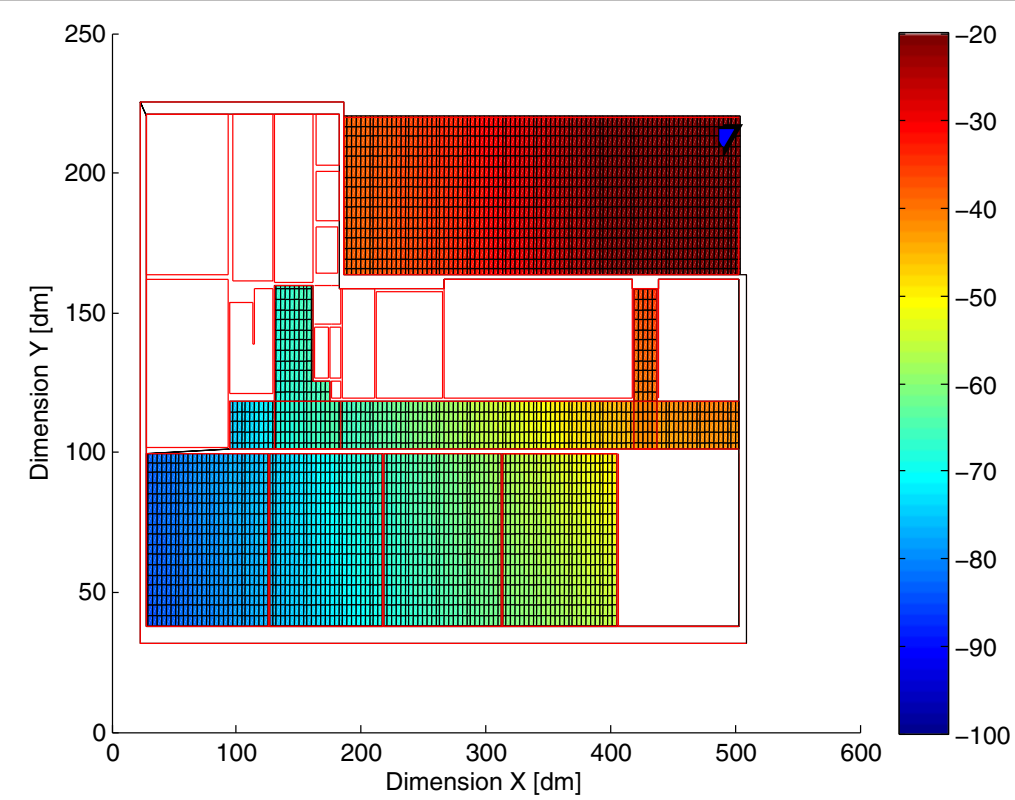

Figure 4 Simulation of DVB-T2-Lite RF signal propagation. The DVB-T2-Lite transmitter is located on the terrace (the blue triangle), and its parameters are described in Table 2. The path loss model was adopted from [28] and extended for the 800-MHz band according to [29] (see Equations 2 and 4). All values are in $\mathrm{dBm}$. 
measured $1 \mathrm{~dB}$ compression of this $\mathrm{PA}$ is $37.9 \mathrm{dBm}$ $(6.2 \mathrm{~W})$, two-tone third-order intermodulation distortion (IMD3) (tone offset $1 \mathrm{MHz}$ ) is better than -38 $\mathrm{dBc}$ at the output power of $1 \mathrm{~W}$, which corresponds to output intercept point (OIP3) $49 \mathrm{dBm}$. Between the PA and the antenna, there is an attenuator in the signal path. It serves as PA protection in the case of antenna switch-off or strong reflections in the antenna near the field. The JFW Industries 50BR-104 N attenuator (JFW Industries, Indianapolis, IN, USA) was used which was set to $0 \mathrm{~dB}$ during measurement. The mentioned nonlinear distortions caused by PA are not considered in our simulation model.

The used antenna is a multi-element Yagi antenna $\left(G_{\max }=15.4 \mathrm{dBi}\right)$ whose horizontal radiation pattern is shown in Figure 2. The feed line for the TV transmitter chain is a coaxial cable RG58 C/U which has a power loss of approximately $0.35 \mathrm{~dB} / \mathrm{m}$ on the tested bandwidth. Attenuation of the auxiliary connection between ' $\mathrm{N}$ ' and 'BNC' connectors is approximately $0.5 \mathrm{~dB} / \mathrm{m}$.

For the LTE system (HeNB), the Sectron AO-ALTEMG5S antenna (Sectron Inc., Ormond Beach, FL, USA) was used. In our case, it was used as an omnidirectional antenna in vertical polarization $(G<3 \mathrm{dBi})$. After setting up the testbed, we moved with the Sefram 7866HD-T2 analyzer (Sefram Instruments and Systems, Saint-Étienne, France) to measure the received TV signal through all measuring points. The same antenna setup was used as is outlined above for LTE downlink. Once again, we spent 2 min at each measurement point for correctly evaluating the performance of the received DVB-T2-Lite RF signal (to avoid fast fading by averaging).

Figures 5 and 6 show measured and extrapolated values of RSS. Figure 5 shows the results of LTE radio signal propagation while Figure 6 shows the results of T2-Lite radio signal propagation obtained from measurement. System parameters determined according to the simulation results proves accessibility of wireless service in all tested locations. As we can see, results from measurement, shown in Figures 5 and 6, correspond with simulation results shown in Figures 3 and 4. This experimental result proves our simulation technique valid for coexistence applications.

Afterwards, the whole measurement campaign was repeated, but now both wireless services (DVB-T2-Lite and LTE) were provided together at the same time. The above outlined QoS parameters of both services, caused by coexistence between them, were measured separately with Rohde \& Schwarz devices.

\section{Experimental results}

5.1 Parameters to evaluate the performance of DVB-T2Lite and LTE

Before evaluating and discussing the obtained results, it is necessary to briefly define the most important measured parameters which were used to evaluate the performance of T2-Lite and LTE systems. To evaluate the quality of the received and decoded TV services, the Quasi Error-Free (QEF) reception conditions were monitored. QEF is a minimal limit defined in the DVB-T2-Lite standard for achieving video service availability without noticeable errors in the video. To fulfill such requirements, the bit error ratio (BER) after FEC decoding must be less than or equal to $1 \times 10^{-7}$ [6].

To evaluate the performance of LTE, the RSS, CQI, and EVM parameters were monitored. The CQI contains information sent from the UE to the HeNB to indicate a suitable downlink transmission data rate. It is based on the observed signal-to-interference-plus-noise ratio (SINR) and used by the HeNB for downlink scheduling and link adaptation [28]. There are 15 different CQI values (numbered from 1 up to 15). The connection between them and the modulation scheme can be found in [35] (Table 7.2.3-1).

EVM, the second parameter, is a measure used to quantify the performance of an LTE communication link. It is the RMS value of the distance in the IQ constellation diagram between the ideal constellation point and the point received by the receiver. For each modulation, there is a defined EVM limit, for which the transmitted signal has an acceptable quality. This limit is equal to $17.5 \%$ for quadrature phase-shift keying (QPSK), $12.5 \%$ for quadrature amplitude modulation (16QAM), and $8.0 \%$ for 64QAM $[11,28]$.

\subsection{DVB-T2-Lite and LTE performance evaluation}

In Subsection 4.1, it has been mentioned that the linear coexistence model maps input parameters from simulations and measurements to the area of QoS states. We have defined the following QoS states for the coexisting services. For DVB-T2-Lite, there are two states: correct reception and no reception. In the case of correct reception, the above defined condition for QEF reception is satisfied. For LTE, we have defined four QoS states which differ in user bitrate and potential radio access network (RAN) throughput. These parameters obviously increase with $\mathrm{M}$ in M-QAM modulation of subcarriers. The LTE system changes the modulation scheme adaptively according to the channel parameters (e.g., CQI, EVM). To be more precise, the highest useable M-state for the defined interfered radio channel sets the QoS state of LTE. Four states correspond to maximal $M$ equaling 64 (64QAM), 16 (16QAM), and 4 (QPSK), and the state when providing LTE services is not possible.

The considered coexistence scenarios between DVB-T2Lite and LTE services were described above. Furthermore, we also consider various system parameters. The complete list of assumed scenarios is clearly summarized in Table 3. There are three main parameters: bandwidth of the LTE RF 


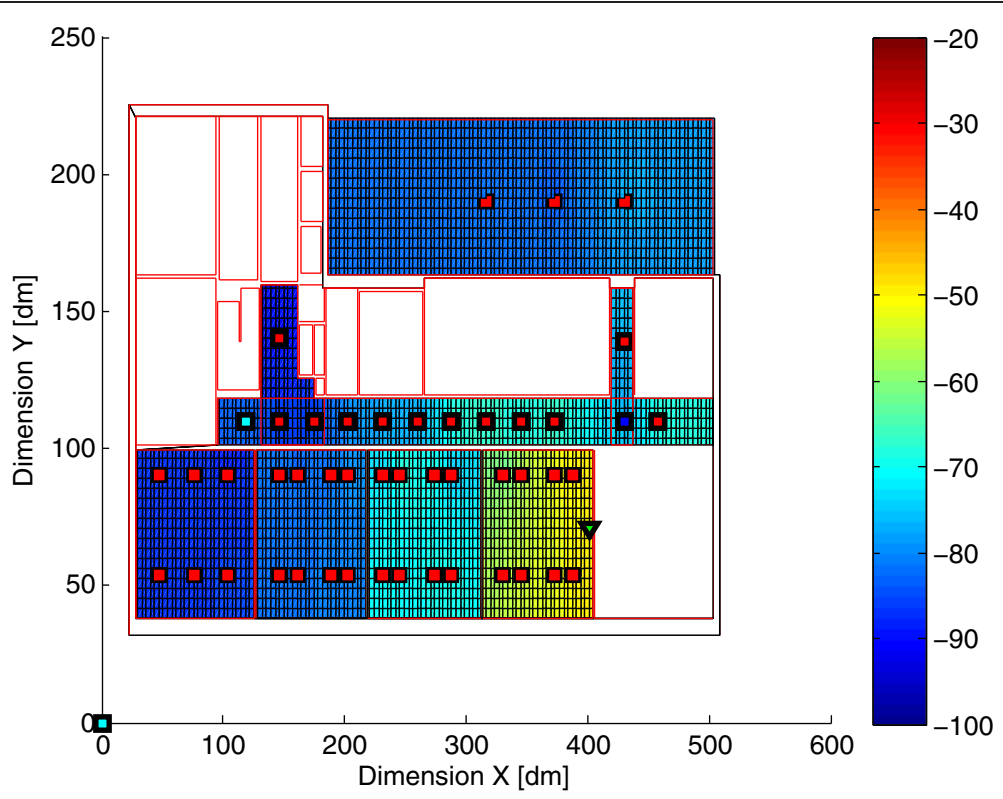

Figure 5 Measurement of LTE RF signal propagation. The HeNB is located in room 7107 (the blue triangle), and network parameters are described in Table 2. The measurement was carried out in highlighted points and extrapolated using MATLAB. All values are in dBm.

channel (marked as $B_{\mathrm{LTE}}$ ), overlap of coexisting channel $\left(B_{\text {OVER }}\right)$, and the power imbalance between transmitted powers $(\Delta P)$.

The last one is calculated as follows:

$$
\Delta P[\mathrm{~dB}]=\mathrm{EIRP}_{\mathrm{LTE}}-\mathrm{EIRP}_{\mathrm{TV}}
$$

where equivalent isotropically radiated power $(\text { EIRP) })_{\mathrm{LTE}}$ and EIRP $_{\mathrm{TV}}$ denote the channel power of LTE and T2Lite RF signals, respectively.

Figure 7 shows the simulated results of six map representations of QoS states in DVB-T2-Lite and LTE systems. Each map (from (a) to (f)) corresponds to the

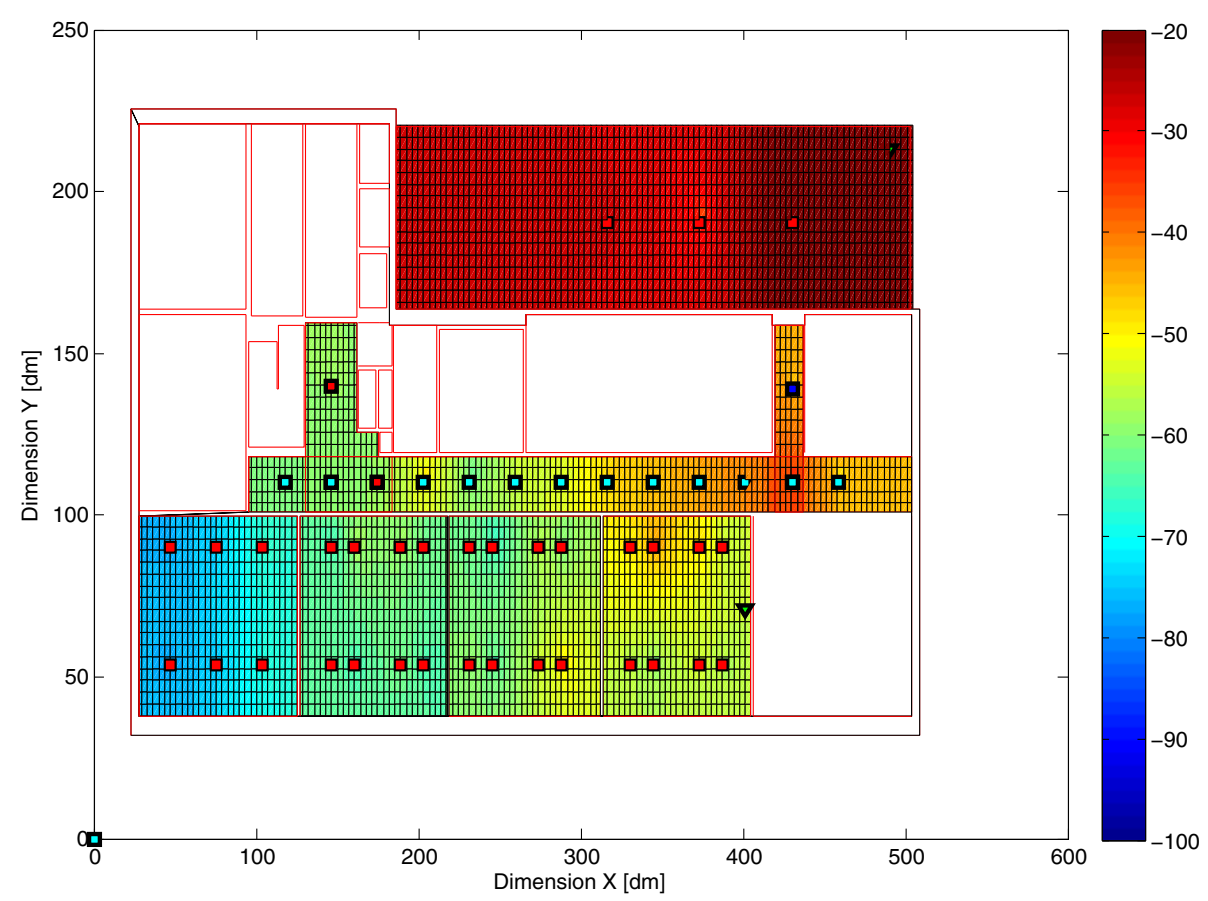

Figure 6 Measurement of DVB-T2-Lite RF signal propagation. The HeNB is located in room 7107 (the blue triangle), and network parameters are described in Table 2. The measurement was carried out in highlighted points and extrapolated using MATLAB. All values are in dBm. 
Table 3 Variable parameters of DVB-T2-Lite and LTE for assumed coexistence scenarios

\begin{tabular}{llll}
\hline Map & $\boldsymbol{B}_{\text {LTE }}(\mathbf{M H z})$ & $\boldsymbol{B}_{\text {OVER }}(\mathbf{k H z})$ & Power imbalance $(\boldsymbol{\Delta} \boldsymbol{P})(\mathbf{d B})$ \\
\hline $\mathrm{a}$ & 10 & 800 & 0 \\
$\mathrm{~b}$ & 20 & 800 & 0 \\
$\mathrm{c}$ & 20 & 1,600 & 0 \\
$\mathrm{~d}$ & 20 & 1,600 & -10 \\
$\mathrm{e}$ & 20 & 1,600 & -20 \\
$\mathrm{f}$ & 20 & 3,000 & 0 \\
\hline
\end{tabular}

considered system parameters and coexistence scenarios which are presented in Table 3. In the floor plan of the university, for each point in the explored areas, the state of both coexisting systems is indicated.

Performances of T2-Lite and LTE systems can be clearly explained in the legend of Figure 7. Four colors represent the LTE maximum useable internal modulations: orange - 64QAM, yellow - 16QAM, and green QPSK, and unavailable LTE services are indicated by a cyan color. The performance of DVB-T2-Lite services is indicated by a crosshatch in the same maps. The presence of a hatch means that the QEF limit of mobile TV reception is fulfilled. For a better explanation of the obtained results, we describe a specific example.

For example, we consider a partial overlapping coexistence scenario between T2-Lite and LTE services when $B_{\mathrm{LTE}}=20 \mathrm{MHz}, B_{\mathrm{OVER}}=1,600 \mathrm{kHz}$, and $\Delta P$ is equal to $0 \mathrm{~dB}$ (see line (c) in Table 3). Performances of coexisting systems for these parameters are plotted in Figure 7c. As can be seen from the legend, at the $1.6-\mathrm{MHz}$ channel overlapping, in the LTE system, only sub-frames using QPSK and 16QAM modulations will be received and demodulated correctly (yellow color in the legend) on the left side of the corridor. It means that only at these modulations EVM errors do not exceed the permitted limit values [11]. In the remaining rooms, the highest 64QAM modulation (highlighted by orange color) is used in the LTE system. Consequently, CQI values can be 10 or higher. Furthermore, this field also indicates that the services of DVB-T2-Lite are highly noised and conditions for QEF reception are not fulfilled (there are no hatched parts). The situation result is the opposite on the terrace where DVB-T2-Lite services are broadcasted. At this place, the provided LTE services are not available (blue color). In this case, the LTE system could not decode the received signal and the CQI value is the lowest. Interestingly, in the small corridor, located between the terrace and the main floor corridor, partial coexistence between T2-Lite and LTE systems is possible. It means that at this place, both wireless systems can coexist. The QEF limit for DVB-T2-Lite is still fulfilled. However, in the LTE system, only sub-frames using QPSK modulation can be successfully processed. Hence, the CQI indicator values will be in the range from 1 up to 6 [35]. Similar graphical representations of considered coexistences are plotted in Figure 7a,b,c,d,e,f.

Now, let us focus on the first two charts (see Figure 7a,b). Their parameters differ just in the used LTE channel bandwidth $\left(B_{\text {OVER }}\right)$, but the disparity in state map is high. From the point of $B_{\mathrm{LTE}}=20 \mathrm{MHz}$ LTE channel (see Figure $7 \mathrm{~b}$ ), the $800 \mathrm{kHz}$ interference bandwidth is quite narrow and almost no effect can be seen on LTE inside the building. Outside, LTE works correctly with 16QAM. However, when $B_{\mathrm{LTE}}$ is equal to $10 \mathrm{MHz}$ (see Figure 7a), then the LTE channel, affected by the same interference bandwidth $(800 \mathrm{kHz})$, is occupied by almost twice the interfering RF power. In this case, the LTE system still works correctly, but only 16QAM and QPSK (indoor/outdoor) modulations can be used. Furthermore, mobile TV reception is also more affected by LTE services because LTE interference power is concentrated into a narrower channel. In real RANs, where power limits are more likely set to $1 \mathrm{~Hz}$ of occupied bandwidth, the impact on the reception of mobile TV services would be the same. The influence of channels overlapping and the effect of different EIRP unbalances could be investigated from the remaining charts.

Figure 8 shows six map representations of QoS states in DVB-T2-Lite and LTE systems from measurements. In general, in most measuring points, the defined states of QoS correspond with simulation results. However, there are some minor differences caused by the accumulation of two types of uncertainties. The first ones are caused by path loss channel modeling, and these are even multiplied by the second ones, caused by the proposed linear model. Most probably, the largest influences are due to inhomogeneity in walls (doors, windows and various types of material), underestimation of noise level, and impact of multipath propagation. It is obvious that the simulation and measurement results in scenario (e) have the lowest difference. This state is caused by the highest signal level (in the above mentioned scenario) which brings reduction of noise background impact and increase the influence of intersystem jamming simultaneously for all transmission paths.

\section{Conclusions}

The main aim of this paper is to investigate the impact of coexisting DVB-T2-Lite and LTE systems in a shared frequency band on their system performances in the outdoor-to-indoor reception scenario. To be more precise, a scenario was considered where an indoor LTE femtocell (HeNB) and outdoor-to-indoor DVB-T2-Lite services are provided in an $800-\mathrm{MHz}$ frequency band (see Figures 1 and 2). We have performed separate simulations of both LTE and DVB-T2-Lite RF signal propagation in MATLAB. Further, we have carried out measurements 


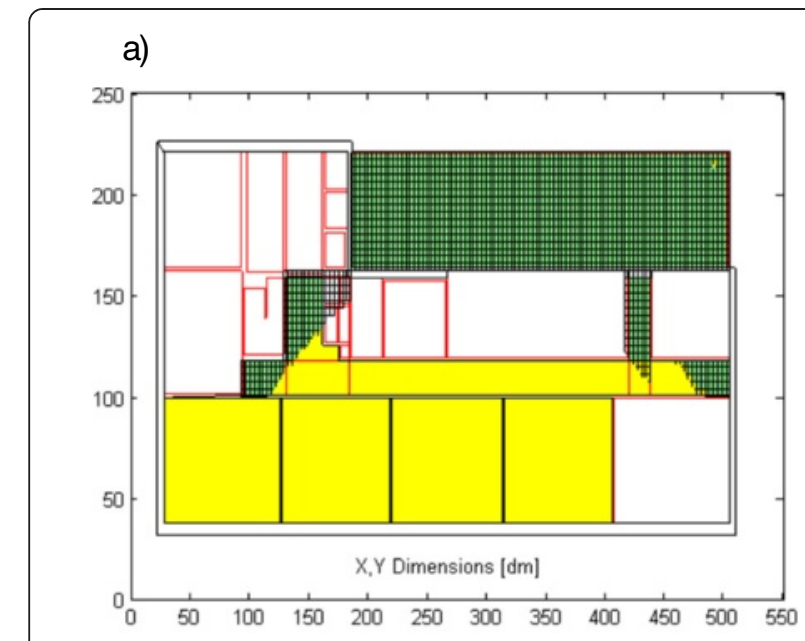

b)

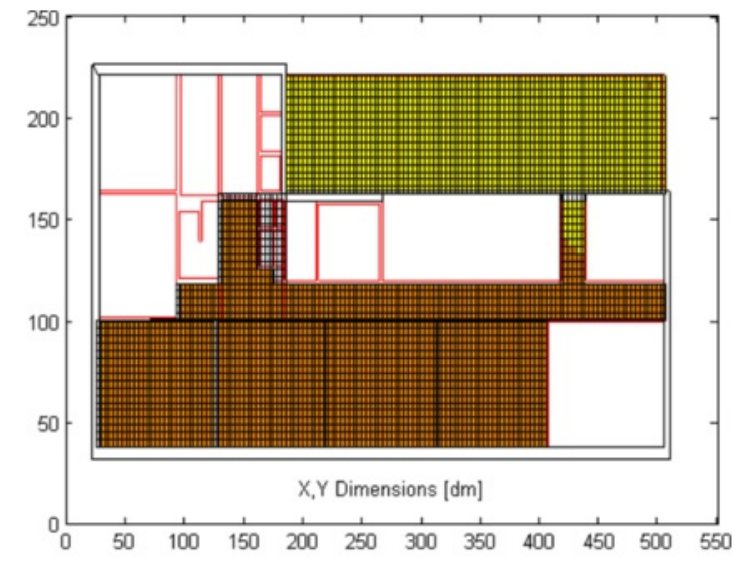

c)
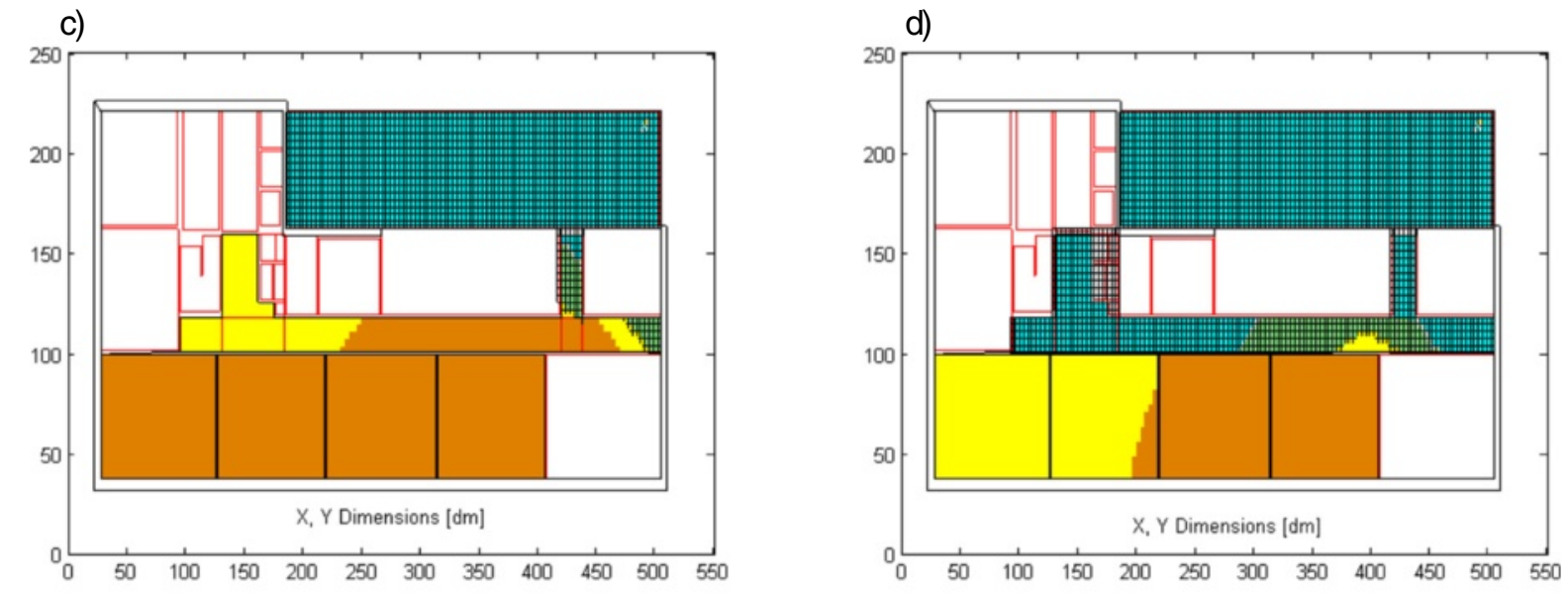

e)

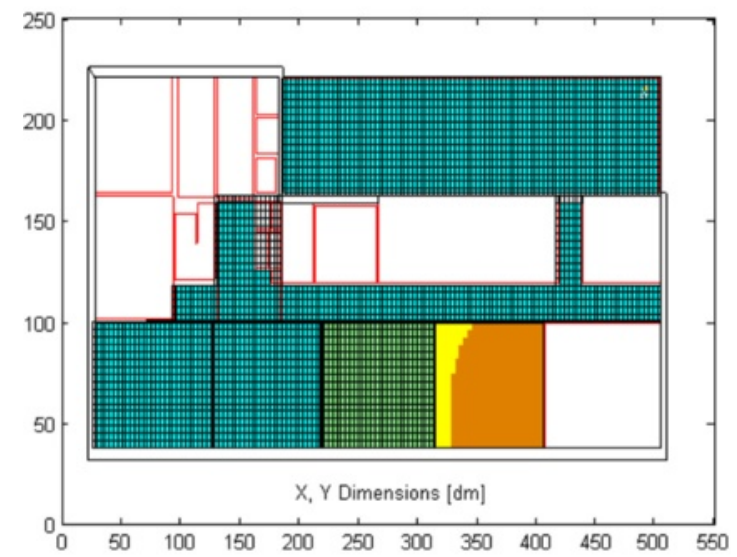

f)
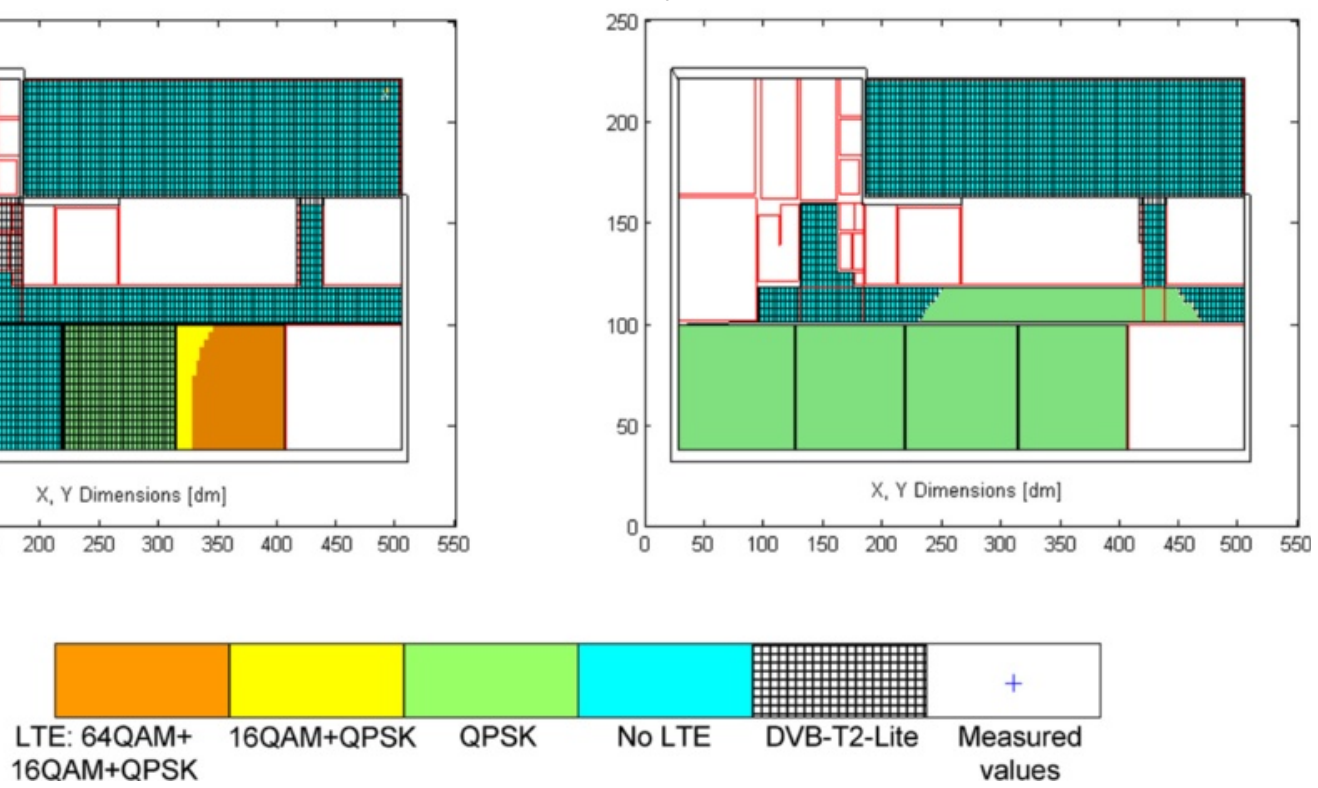

Figure 7 Simulation - the map representation of QoS states of coexisting systems (a-f). Specific map parameters are summarized in Table 3. 
a)

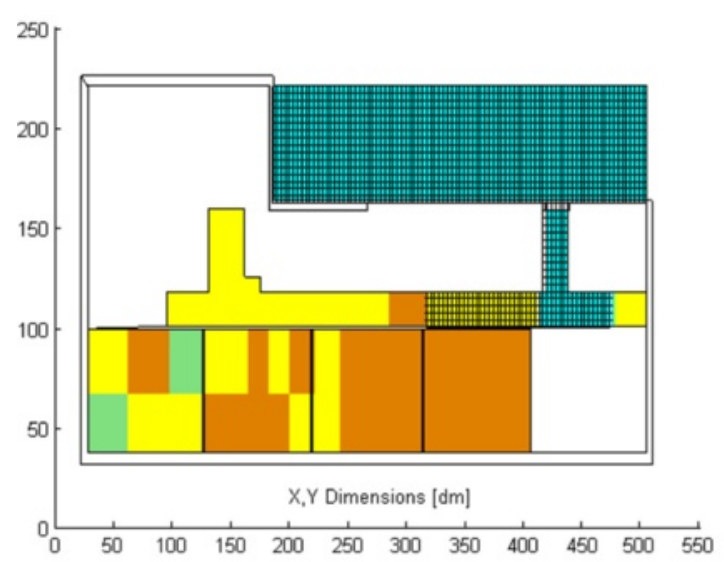

c)

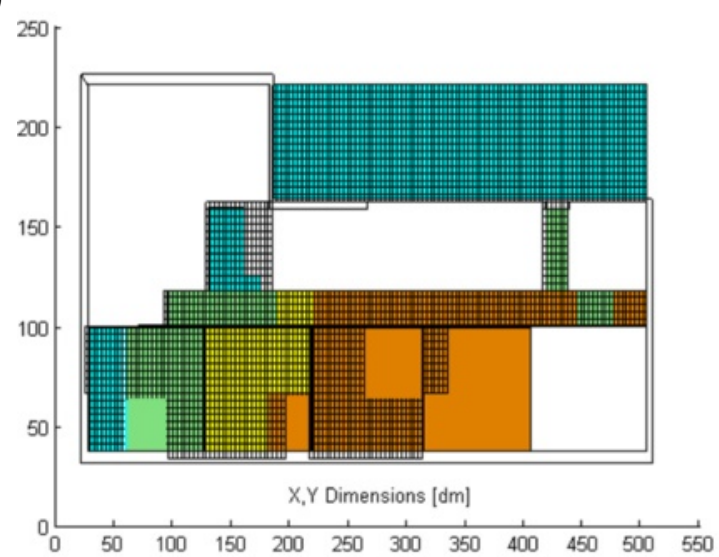

e)

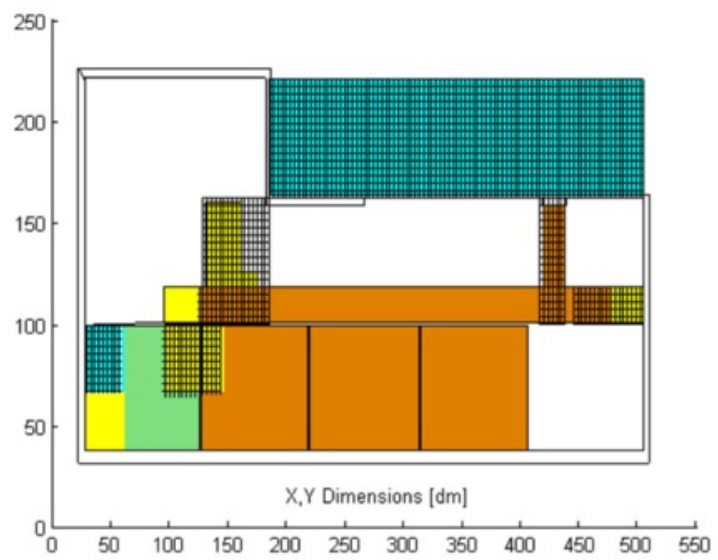

b)

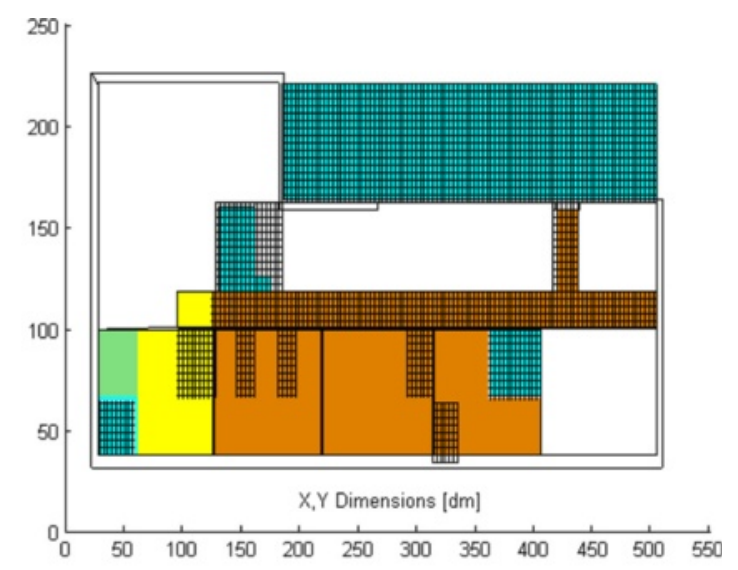

d)

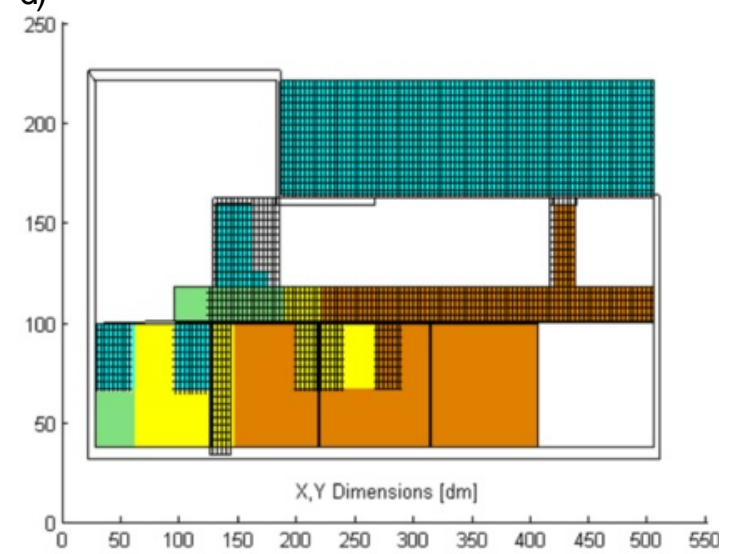

f)

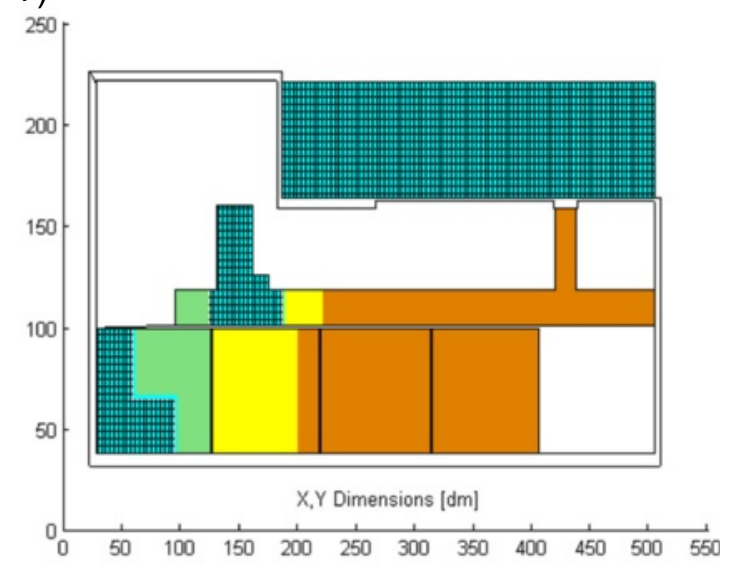

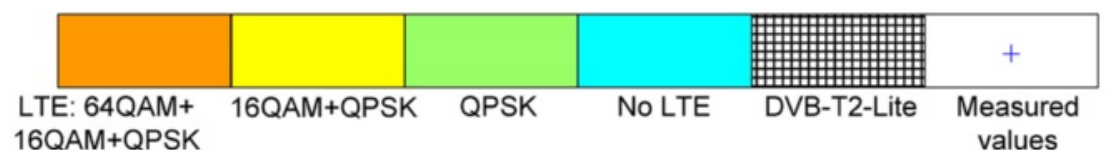

Figure 8 Measurement - the map representation of QoS states of coexisting systems (a-f). Specific map parameters are summarized in Table 3. 
of both wireless systems in order to evaluate the reliability of the simulation model. Results are shown in Figures 3, 4, 5,6 and correlate well.

According to the achieved results in our previous works ([31] and [28]), we have created a linear model to map outputs of the path loss model to defined QoS states. This model considers the relation between the value of RF channels overlapping and the power imbalance of the investigated radio channels. A detailed description is outlined in Subsection 4.1.

The presented results are expressed in a set of maps (floor plans of the building) with colored areas which determine availability or non-availability of coexisting services and achievable performance. Specific values of these parameters in the considered scenarios are presented in Table 3. The effect of coexistence on valid signal reception is quantified by the change of used modulation scheme and simultaneous availability of services. A detailed description of the color maps is described in Section 5. In the proposed linear model for both systems, we assume good channel conditions (global parameters): signal-to-noise ratio (SNR) $\geq 35 \mathrm{~dB}$ for both systems and also no multipath propagation and no Doppler frequency have been set. Once again, the proposed linear model was proved by measurements (see Figures 7 and 8). In several cases, less correspondence between the simulation and measurement results is explained.

An analysis of the obtained results from the considered coexistence scenarios leads to the following general conclusions:

a) The impact of DVB-T2-Lite system performance on the LTE system performance and vice versa in their co-channel coexistence scenario in a shared frequency band highly depends on the level of their channels overlapping and on the power imbalance between RF signals.

b) The outdoor-to-indoor penetration of the T2-Lite signal is highly critical on indoor-to-indoor reception of LTE services when the power imbalance between the RF levels is high. In these cases, the T2-system acts as a co-channel interferer to indoor LTE femtocell and vice versa.

c) Digital TV fixed indoor reception is more vulnerable to interferences than fixed outdoor reception.

The main aim of our future work will be to extend our proposed linear coexistence model with more global parameters (different kinds of fading channel models and Doppler shift [36-39]) for more realistic modeling of different coexistence scenarios between DVB-T2-Lite and LTE services and vice versa. Moreover, in our future work, we will consider a larger range of system parameters (code rate, IFFT length, guard interval, and higher M-QAM modulations and bandwidth) $[40,41]$.

\section{Competing interests}

The authors declare that they have no competing interests.

\section{Acknowledgement}

This work is supported by the Cluster for Application and Technology Research in Europe on Nanoelectronics (CATRENE) under the project named CORTIF CA116 - Coexistence of Radio Frequency Transmission in the Future, the MEYS of the Czech Republic no. LF14033, no. CZ.1.07/2.3.00/20.0007 and CZ.1.07/2.3.00/30.0005, and finally by the BUT project no. FEKT-S-14-2177. The described research was performed in laboratories supported by the SIX project; no. CZ.1.05/2.1.00/03.0072, the operational program Research and Development for Innovation. Research described in this paper was financed by Czech Ministry of Education in frame of National Sustainability Program under grant LO1401. For research, infrastructure of the SIX Center was used.

Received: 31 October 2014 Accepted: 24 March 2015

Published online: 22 April 2015

\section{References}

1. Z Raida et al., Communication subsystems for emerging wireless technologies. Radioengineering 21(4), 1036-1049 (2012)

2. T Yos, Y Wu, N Hur, T Ikeda, P Xia, FOBTV: worldwide efforts in developing next-generation broadcasting system. IEEE Trans. on Broadcasting 60(2), 154-159 (2014)

3. L Klozar, L Polak, O Kaller, J Prokopec, Effect of Co-Existence Interferences on QOS of HSPA/WCDMA Mobile Networks, in Proceedings of the 23rd IEEE International Conference Radioelektronika 2013, 2013, pp. 312-315

4. W Sami, How can Mobile and Broadcasting Networks use Adjacent Bands? in EBU Technical Review, 2011, pp. 1-20

5. Digital Video Broadcasting (DVB), Framing Structure, Channel Coding, and Modulation for Digital Terrestrial Television Broadcasting System (DVB-T2), 2012. ETSI EN 302755 V1.3.1

6. Digital Video Broadcasting (DVB), Implementation Guidelines for a Second Generation Digital Terrestrial Television Broadcasting System (DVB-T2), 2012. 2012. ETSI TS 102831 V1.2.1

7. ITU-Rec, Frequency and Network Planning Aspects of DVB-T2, 2012. ITU-R BT.2254

8. DA Samo, M Slimani, G Baruffa, L Rugini, A performance study of DVB-T2 and DVB-T2-Lite for mobile reception. Digital Signal Processing 37(2), 35-42 (2015)

9. C Regueiro, U Gil, M Velez, I Eizmendi, P Angueira, Field trials-based planning parameters for DVB-T2 indoor reception. IEEE Trans on Broadcasting. (2015). doi:10.1109/TBC.2015.2400814)

10. Digital Video Broadcasting (DVB), Framing Structure, Channel Coding and Modulation for Digital Terrestrial Television, 2009. ETSI EN 300744 v1.6.1

11. 3GPP, 3rd Generation Partnership Project, Evolved Universal Terrestrial Radio Access (E-UTRA); User Equipment (UE) Radio Transmission and Reception (Release 8), 2010. Tech. Specification 3GPP TS 136.101 V8.9.0

12. E Dahlman, 3G Evolution: HSPA and LTE for Mobile Broadband, 2nd edn. (Academic Press, Oxford, 2008)

13. D Astely, E Dahlman, A Furuskar, Y Jading, M Lindstrom, S Parkvall, LTE: the evolution of mobile broadband. IEEE Communication Magazine 47(4), 44-51 (2009)

14. Rohde \& Schwarz, Coexistence digital TV and LTE (Rohde \& Schwarz, 2012), http://cdn.rohde-schwarz.com/pws/dl_downloads/dl_application/ application_notes/1ma176/1MA176_3e_coexistence_digital_TV_and_LTE.pdf

15. P Stavroulakis, Interference Analysis and Reduction for Wireless Systems (Artech House, Norwood, 2003)

16. F Gleissner, S Hanus, Co-channel and adjacent channel interference measurement of UMTS and GSM/EDGE systems in $900 \mathrm{MHz}$ radio band. Radioengineering 17(3), 74-80 (2008)

17. J Mikulka, S Hanus, Bluetooth and IEEE $802.11 \mathrm{~b} / \mathrm{g}$ coexistence simulation. Radioengineering 17(3), 66-73 (2008)

18. B Han, W Wang, Y Li, M Peng, Investigation of interference margin for the co-existence of macrocell and femtocell in orthogonal frequency division multiple access systems. IEEE Systems Journal 7(1), 59-67 (2013) 
19. A Lourerio, D Callegos, G Caldwell, Interference Analysis on UMTS-2100 Co-Existence with GSM-1900, in Proceedings of the 74th IEEE VTC FALL 2011, 2009, pp. 1-4

20. WA Hassan, TA Rahman, Coexistence model for compatibility between IMT-advanced and other wireless communication services. Wireless Personal Communications 79(3), 2025-2039 (2014)

21. MH Ng, S-D Lin, J Li, S Tatesh, Coexistence studies for 3GPP LTE with other mobile systems. IEEE Communications Magazine 47(4), 60-65 (2009)

22. D Lee, GY Li, S Tang. Inter-Cell Interference Coordination for LTE Systems, in IEEE GLOBECOM 2012, 4828-4833 (2012)

23. T Cai, J Deng. Coexistence Study and Interference Analysis in LTE Networks, in IEEE International Conference ICCECT 2012, 751-754 (2012)

24. L Tytgat, O Yaron, S Pollin, I Moerman, P Demeester, Avoiding collisions between IEEE 802.11 and IEEE 802.15.4 through coexistence aware clear channel assessment. EURASIP Journal on Wireless Communications and Networking 2012, 137 (2012)

25. M Boujelben, S Benrejeb, S Tabbane, A Comparative Study of Interference Coordination Schemes for Wireless Mobile Advanced Systems, in The 2014 International Networks, Computers and Communications Symposium (ICNC 2014), 2014, pp. 1-5

26. D Chen, T Jiang, Z Zhang, Frequency partitioning methods to mitigate crosstier interference in two-tier femtocell networks. IEEE Trans on Vehicular Technology, 2015. doi:10.1109/TVT.2014.2335251

27. ZA Shamsan, LTE-Advanced Compatibility with Digital Broadcasting Receiver at $800 \mathrm{MHz}$, in 2013 Saudi International Electronics, Communications and Photonics Conference (SIECPC), 2013, pp. 1-4

28. 3GPP, 3rd generation partnership project, Technical specification group radio access network; evolved universal terrestrial radio access (E-UTRA); further advancements for E-UTRA physical layer aspects (Release 9), March 2010. Tech. Report 3GPP TR 36.814 V9.0.0, http://www.qtc.jp/3GPP/Specs/ 36814-900.pdf

29. 3GPP, 3rd generation partnership project, Technical specification group radio access network; evolved universal terrestrial radio access (E-UTRA); study on LTE device to device proximity services-radio aspects (Release 12), November 2013. Tech. Report 3GPP TR 36.843 V12 .0.0, http:// www.3gpp.org/dynareport/36843.htm

30. L Polak, O Kaller, L Klozar, J Prokopec, Exploring and Measuring the CoExistence Between LTE and DVB-T2-Lite Services, in Proceedings of the 36th International Conference on Telecommunications and Signal Processing (TSP) 2013, 2013, pp. 316-320

31. L Polak, O Kaller, L Klozar, J Sebesta, T Kratochvil, Mobile communication networks and digital television broadcasting systems in the same frequency bands - advanced co-existence scenarios. Radioengineering 23(1), 375-386 (2014)

32. Software package Amari LTE. Amari LTE 100/Amari OTS 100 Software 4G network on PC. Online on: < http://www.amarisoft.com/?p=amarilte>

33. M Slanina, L Klozar, S Hanus, Practical Measurement of Data Throughput in LTE Network Depending on Physical Layer Parameters, in Proceedings of the 24th International Conference Radioelektronika, 2014, pp. 1-4

34. RA20H8087M. RF MOSFET module 806-870 MHz 20 W 12.5 V, 3 Stage Amplifier for Mobile Radio. Data Sheet. Mitsubishi Electric Corporation July 2011, http://datasheet.eeworld.com.cn/pdf/MITSUBISHI/ 180155_RA20H8087M.pdf

35. 3GPP, 3rd generation partnership project, Technical specification LTE; evolved universal terrestrial radio access (E-UTRA); physical layer procedures (Release 8), October 2009. Tech. Specification 3GPP TS 136213 V8.8.0, http://www.3gpp.org/dynareport/36213.htm

36. J Milos, S Hanus, Performance analysis of PCFICH and PDCCH LTE control channels. Radioengineering 23(1), 445-451 (2014)

37. L Polak, T Kratochvil, Analysis and simulation of the transmission distortions of the mobile digital television DVB-SH part 2: satellite mode DVB-SH-B with TDM. Radioengineering 21(1), 126-133 (2012)

38. L Polak, T Kratochvil, Exploring of the DVB-T/T2 Performance in Advanced Mobile TV Fading Channels, in Proceedings of the 36th International Conference on Telecommunications and Signal Processing (TSP) 2013, 2013, pp. 768-772

39. L Polak, O Kaller, L Klozar, J Sebesta, T Kratochvil, Exploring and measuring possible co-existences between DVB-T2-lite and LTE systems in ideal and portable fading channels. Journal of Applied Research and Technology 13(1), 32-44 (2015)
40. H Hong, H Son, Y Chong, Protection Ratio Between DVB-T2 and LTE System, in 2014 Information and Communication Technology Convergence (ICTC), 2014, pp. 792-793

41. M Fuentes, C Garcia-Pardo, E Garro, D Gomez-Barquero, N Cardona, Coexistence of digital terrestrial television and next generation cellular networks in the $700 \mathrm{MHz}$ band. IEEE Wireless Communications 21(6), 63-69 (2014)

42. A Guidotti, D Guiducci, M Barbiroli, C Carciofi, P Grazioso, G Riva, Coexistence and Mutual Interference Between Mobile and Broadcasting Systems, in Proceedings of the 73rd IEEE Vehicular Technology Conference (VTC Spring), 2011, pp. 1-5

43. K Sakic, S Grgic, The Influence of the LTE System on the DVB-T Reception, in Proceedings of the 52nd International Symposium ELMAR, 2010, pp. 235-238

44. G Baruffa, M Femminella, F Mariani, G Reali, Protection ratio and antenna separation for DVB-T/LTE coexistence issues. IEEE Communications Letters 17(8), 1588-1591 (2013)

45. H Bawab, P Mary, J-F Hélard, YJ Nasser, O Bazzi, Global Ergodic Capacity Closed-Form Expression of Coexisting DVB-LTE-Like Systems, in 2014 IEEE VTC Spring, 2014, pp. 1-5

46. A Tekovic, G Simac, K Sakic, LTE Downlink System Performance Measurement with Intersystem Interference Caused by DVB-T Signal, in Proceedings of the 54th International Symposium ELMAR, 2012, pp. $255-258$

47. A De Vita, D Milanesio, B Sacco, A Scotti, Assessment of interference to the DTT service generated by LTE signals on existing head amplifiers of collective distribution systems: a real case study. IEEE Trans. on Broadcasting 60(2), 420-429 (2014)

48. L Polak, O Kaller, L Klozar, J Prokopec, Influence of Mobile Network Interfering Products on DVB-T/H Broadcasting Services, in Proceedings of the 5th IEEE International Conference Wireless Days 2012, 2012, pp. 1-5

\section{Submit your manuscript to a SpringerOpen ${ }^{\odot}$ journal and benefit from:}

- Convenient online submission

Rigorous peer review

- Immediate publication on acceptance

- Open access: articles freely available online

- High visibility within the field

- Retaining the copyright to your article

Submit your next manuscript at $>$ springeropen.com 